\title{
Novel Molecular Biomarkers at the Blood-Brain Barrier in ALS
}

\author{
Danijela Bataveljic, Milena Milosevic, Lidija Radenovic, and Pavle Andjus \\ Center for Laser Microscopy, Institute for Physiology and Biochemistry, Faculty of Biology, University of Belgrade, \\ Studentski Trg 16, POB 52, 11000 Belgrade, Serbia \\ Correspondence should be addressed to Lidija Radenovic; lidijar@bio.bg.ac.rs
}

Received 14 February 2014; Revised 4 April 2014; Accepted 20 April 2014; Published 11 May 2014

Academic Editor: Raquel Manzano

Copyright (c) 2014 Danijela Bataveljic et al. This is an open access article distributed under the Creative Commons Attribution License, which permits unrestricted use, distribution, and reproduction in any medium, provided the original work is properly cited.

Recently neuroinflammation has gained a particular focus as a key mechanism of ALS. Several studiesin vivo as well asin vitro have nominated immunoglobulin G (IgG) isolated from ALS patients as an active contributor to disease onset and progression. We have shown that ALS IgG affects astroglial $\mathrm{Ca}^{2+}$ excitability and induces downstream activation of phosphatidylinositol 3-kinase. These studies were hampered by a lack of knowledge of the pathway of entry of immune factors in the CNS. Our MRI data revealed the blood-brain barrier BBB leakage and T cell infiltration into brain parenchyma in ALS G93A rats. Since astrocyte ensheathes blood vessel wall contributing to BBB stability and plays an important role in ALS pathogenesis, we have studied astrocytic membrane proteins water channel aquaporin-4 and the inwardly rectifying potassium channel. In this review, we will summarize data related to BBB disruption with particular emphasis on impaired function of astrocytes in ALS. We will discuss implication of membrane proteins expressed on astrocytic endfeet, aquaporin-4, and inwardly rectifying potassium channel in the pathology of ALS. In addition to ALS-specific IgGs, these membrane proteins are proposed as novel biomarkers of the disease.

\section{Overview}

Amyotrophic lateral sclerosis (ALS) is an adult-onset, progressive neurodegenerative disease that affects upper and lower motor neurons leading to fatal paralysis mostly within a few years. This devastating disease was described back in the 1870s, but despite the progress in biomedicine, no suitable therapeutic treatment has been introduced. Moreover, there is no distinguishable diagnostic test for the disease. Riluzole, a substance with antiglutamatergic properties, which modestly extends survival by $11 \%$ [1], remains the only approved drug for the treatment of ALS. The majority of research on ALS patients involves investigation of postmortem tissue samples. Development of in vivo approaches broadens the possibility to improve clinical research. In addition, identification of several different mutations that lead to ALS symptoms contributed to the generation of transgenic animal models widely used to examine mechanisms of ALS pathology [2-5].

ALS is in fact a complex disease with several proposed mechanisms, all of which lead to specific degeneration of motor neurons and the dysfunction of the neuromuscular junction. As one of the key mechanisms underlying the etiopathogenesis of ALS, glutamate excitotoxicity and its implications have been widely addressed and reviewed elsewhere. Here, we would like to point out some key features of brainstem and spinal motor neurons, important for their normal functioning that make them more susceptible in the elevated brain activity. Namely, ALS-vulnerable motor neurons have lower capacity for calcium buffering with larger calcium microdomains in the vicinity of open channels and high number of calcium permeable AMPA receptors. Importantly, these neurons depend considerably on surrounding astrocytes that are able to influence the expression level of the calcium impermeable GluR2 subunit of AMPA receptors and are responsible for glutamate clearance and potassium buffering (for reviews see [610]). However, there is a wealth of literature on aberrant populations of astrocytes in ALS. Astrocytes in ALS are known to change shape and swell $[11,12]$ that is indicative of their hampered extracellular ion filtering function [13]. On the other hand, there is evidence that in ALS astrocytes 
release toxic factors that are detrimental to motor neurons [14-18].

\section{Neuroinflammation: A Focus on Humoral Immunity}

Cellular markers of neuroinflammation in ALS (activated astrocytes and microglia, followed by infiltrated Tlymphocytes) are considered to be one of the main characteristics of ALS, as shown in numerous studies on human postmortem samples and on animal models of the disease (for reviews see [19-21]). All these findings suggest that neuroinflammation in ALS is not a temporary, but rather permanent, feature. The activation of the immune response, although beneficial at start, enters the vicious cycle that contributes to further neuronal damage, due to continuous presence of inflammatory cause, thus creating the inability to end the inflammatory reaction [19].

Over the last four decades, many attempts have been made to recognize useful biomarkers for ALS in biofluids. A concept of combining markers to improve accuracy has been widely supported, where some candidate biomarkers may be useful to aid in the diagnostics but may also have better applications in monitoring drug effects in clinical trials and as prognostic indicators of disease (for review see [22]). Here, we will focus on possible humoral immune markers, mainly circulating immune complexes (CICs) and immunoglobulins (Ig).

Several studies have demonstrated elevated CICs in ALS patients [23-26], but Saleh and coworkers (2009) were able to evaluate humoral immune response over disease progression by following the group of ALS patients in a 6-month period. They found elevated CICs as well as IgG in ALS sera in the initial disease stage, but, 6 months after the first tests, the same group of ALS patients, now with lower ALS functional rating score, had CICs lowered to the control levels, yet IgG remained higher than in controls [27]. Regarding the levels of IgG in ALS sera, literature data differ. Some report elevated IgG and normal IgM [26-29] with a change in subclasses [30] or without it [31], while others detect no change [25] or even decrease [32]. Inconsistency of reports is probably due to different selection criteria for ALS patients that were in different phases of the disease.

\section{Specificity and (Patho)physiological Effects of ALS IgG}

More than 20 years ago, Appel and coworkers showed that intraperitoneal injection of IgG purified from sera of sporadic ALS patients (ALS IgG) in mice increased the frequency of miniature end-plate potentials, without changing their amplitude, leading to increased acetylcholine release from motor nerve endings [33]. In addition, IgGs were detected inside motor neurons, as well as in end-plates. Taken together, ALS IgGs were able to elicit physiological abnormalities of nerve-muscle synapse by passive transfer, which indicated possible immune-mediated mechanisms in the pathogenesis of ALS. Passive transfer of ALS IgG in mice caused degeneration of motor neurons and increased proportion of calcium containing organelles [34]. In cell culture preparations, ALS IgG induced apoptotic cell death of the hybrid motoneuronal cell line VSC4.1 [35] and of the human neuroblastoma cell line [36]. In the rat mixed primary spinal cord cultures, ALS IgG activated caspase- 3 and downstream signaling pathways, leading to selective apoptosis of neurons, while astrocytes were less susceptible; hence, the authors left an open possibility that neuronal death could be a secondary effect of the influence of ALS IgG on astrocytes [37].

Treatment of rodents (animals or isolated neuronal preparations) with ALS IgG caused increase in intracellular calcium concentration and degenerative structural changes as well as plasticity induction in the neuromuscular junction [34, 38-42]. Electrophysiological recordings of voltage-gated calcium currents showed an ALS IgG-induced increase in Purkinje neurons [43], but a decrease in cultured granular cells [44]. We have shown that ALS IgG induced the frequency increase, without amplitude changes of spontaneous miniature excitatory postsynaptic currents in primary rat hippocampal pyramidal neurons, and this effect was partially dependent on extracellular calcium [45]. Moreover, ALS IgG could modulate calcium transients in the same model system - an effect that was shown to be dependent on P/Qtype calcium channels [46].

In the recent study of Gonzalez and coworkers (2011) [47], a substantial evidence was presented in favor of the specificity of ALS IgG, as they addressed the distribution of autoantibodies via immunostaining in cerebellar, cortical, and spinal cord and neuromuscular junction sections of mice in addition to ALS IgG-induced physiological response via potentiating effect on end-plate basal discharge activity of neuromuscular junctions. Moreover, the use of $\mathrm{Ca}_{\mathrm{V}} 2.1$ knockout mice allowed them to distinguish between possible direct effect of ALS IgG on P/Q-type calcium channel (as congruent to our work; see above and [46]) and definite indirect (probably via phospholipase C) effect on N-type calcium channels [47].

The role of glial cells and their possible responses to ALS IgG was questioned in the study of the effect of these autoantibodies on motoneuronal morphology and survival in organotypic rat spinal cord cultures, where a range of concentrations of ALS IgG failed to induce significant changes [48]. Moreover, a brilliant cell-type sensitive genetic manipulation of the mouse model of ALS, that underlines the importance of neuron-glia interactions, where astrocytes and microglia are found to be implicated in the rate of progression of familial ALS, reported that the damage in astrocytes determines the timing of microglial activation and infiltration $[49,50]$. Finally, our recent pioneer studies on ALS IgG-induced (patho)physiological changes in cultured rat cortical astrocytes detected specific acute astrocytic responses. Namely, the majority of ALS IgGs increased in a matter of minutes the mobility of acidic vesicles, mostly endosomes and lysosomes, which could suggest long-term modulation of endocytosis and/or autophagy [51]. In addition, half of ALS IgGs tested evoked transient increases in intracellular calcium, mediated via inositol-3-phosphate 
receptors in the endoplasmic reticulum, where the influx of calcium ions through store-operated calcium entry did not affect the amplitude but prolonged calcium transients, thus augmenting the overall magnitude of the response [52]. Elevated calcium could trigger the release of glutamate from astrocytes into the extracellular space via vesicular exocytosis [53], that could contribute to the excitotoxic loss of motor neurons. Although pharmacological analysis revealed activation of phosphatidylinositol 3-kinase upstream of phospholipase C, a direct ALS IgG target on astrocytes remains still elusive. The interaction of ALS-specific antibodies with an unknown astrocytic membrane structure, mediated via phosphatidylinositol 3-kinase that integrates pathways of cell growth, proliferation, differentiation, motility, survival, and intracellular trafficking and can interact with around 50 Src homology domains containing cellular enzymes [54], could have a great impact on etiopathogenesis of ALS, thus stressing out the urge for further investigations. On the other hand, these immune humoral factors also offer a possible new biomarker for ALS diagnostics. Nevertheless, although deposits of IgG were detected in postmortem spinal cords of ALS patients [55], until recently studies involving the interaction of disease specific antibodies and cells of immunologically privileged tissues were hampered by a lack of knowledge of the pathway of entry of immune factors in the CNS, particularly of the blood-brain (BBB) and the blood-spinal cord (BSCB) barrier that will be discussed in the following sections.

\section{Blood-Spinal Cord and Blood-Brain Barrier Damage in ALS}

First indications of disturbed permeability of BSCB and BBB in ALS patients came with the studies that examined blood and cerebrospinal fluid (CSF) composition in ALS patients. Thus, several immunological molecular markers of the BBB compromise were detected in blood and CSF [26]. More recent studies introduced several approaches for investigating BSCB and BBB condition in animal models of ALS, particularly in the regions of motor neuron degeneration. Intravenous application of Evans blue showed vascular leakage in spinal cord of end stage ALS animals [11, 12]. In ALS mice, permeability of vessels for Evans blue was observed even in early stage of disease [11]. Further examination was directed towards endothelial cells and their crucial role in control of transport across BSCB and BBB. Expression of tight junction proteins of endothelial cells, zonula occludens and occludin, was decreased in the spinal cord of ALS mice and rats $[12,56]$. Investigation of $\mathrm{BSCB}$ and $\mathrm{BBB}$ ultrastructure using electron microscope showed swollen and degenerating endothelial cells and capillary rupture in early and end stage ALS mice [57]. Study of BSCB and BBB condition in the postmortem tissue of sporadic ALS patients confirmed findings on animal models [58]. These findings verified damage of endothelial cells and reduced expression of tight junction proteins and vascular leakage of IgG [58].

In order to explore the condition of $\mathrm{BBB}$ in vivo, the experiments on ALS rat brain were conducted using $1.5 \mathrm{~T}$ magnetic resonance imager with surface coil. Application of gadolinium-DTPA contrast marker revealed increased BBB permeability in the brain of symptomatic ALS rat [21]. Simultaneously, antibodies against CD4+ and CD8+ receptors specifically labeled with ultrasmall superparamagnetic iron oxide particles (USPIO) were injected in order to follow infiltration of T-lymphocytes into the rat brain tissue using MRI. Accumulation of USPIO markers was detected in the tissue area surrounding lateral ventricles of the ALS rat. It was suggested that the infiltration of inflammatory cells in the ALS rat brain could be carried through the impaired BBB. A model proposed by Zlokovic (2008) [59] also indicated the $\mathrm{BBB}$ disruption as a crucial event in ALS pathogenesis. Based on the above studies, impairment of BBB opens the way for the entrance of inflammatory cells and factors into the CNS and leads to the activation of astrocytes and microglia and production of reactive oxygen species. These findings were also in favour of the studies of our group and others that showed the effect of ALS IgG on neurons and glia. Thus a hampered $\mathrm{BBB}$ or $\mathrm{BSCB}$ could offer the necessary pathway of entry for these immune factors (see above). Finally, this sequence of changes in the brain would result in a loss of function and death of motor neurons.

\section{Involvement of Astrocytic Proteins in Blood-Brain Barrier Impairment}

Although the most important components of $\mathrm{BBB}$ are endothelial cells, astrocytes contribute to $\mathrm{BBB}$ stability through perivascular endfeet ensheathing blood vessel wall. Moreover, astrocytic endfeet closely surround neuronal synapses contributing to the stability of neuronal microenvironment. Astrocytes express several proteins anchored in the membrane of their endfeet such as water channel aquaporin4 (AQP4) and inwardly rectifying potassium channel Kir4.1 $[60,61]$. These two transmembrane proteins colocalize and play a key role in the maintenance of water and ion homeostasis at the level of BBB and neuronal synapses [62, 63].

As astrocytes have an important role in the $\mathrm{BBB}$ maintenance, their involvement in molecular mechanisms underlying BBB disruption in ALS has been more thoroughly explored (Figure 1). Previous studies on animal models of ALS showed that astrocytic endfeet ensheathing the vessel wall appeared swollen indicating disturbance of water homeostasis and possible involvement of AQP4 [11, 12]. Our MRI investigations of the rat brain at end stage of ALS showed enlargement of lateral ventricles that further suggested impaired function of AQP4 [64]. For that reason, AQP4 expression was examined in several CNS regions affected in ALS. Recent studies have shown that AQP4 was overexpressed in the spinal cord, brainstem, and cortex of ALS rat $[65,66]$. We have also documented that an increased expression of AQP4 was apparent in the astrocytic endfeet, particularly in those surrounding blood vessels [65]. Additionally, astrocytic endfeet that form glia limitans at the brain surface showed increased level of AQP4. Conversely, examination of Kir4.1 expression revealed a decrease in the spinal cord, brainstem, and cortex in ALS animal models 


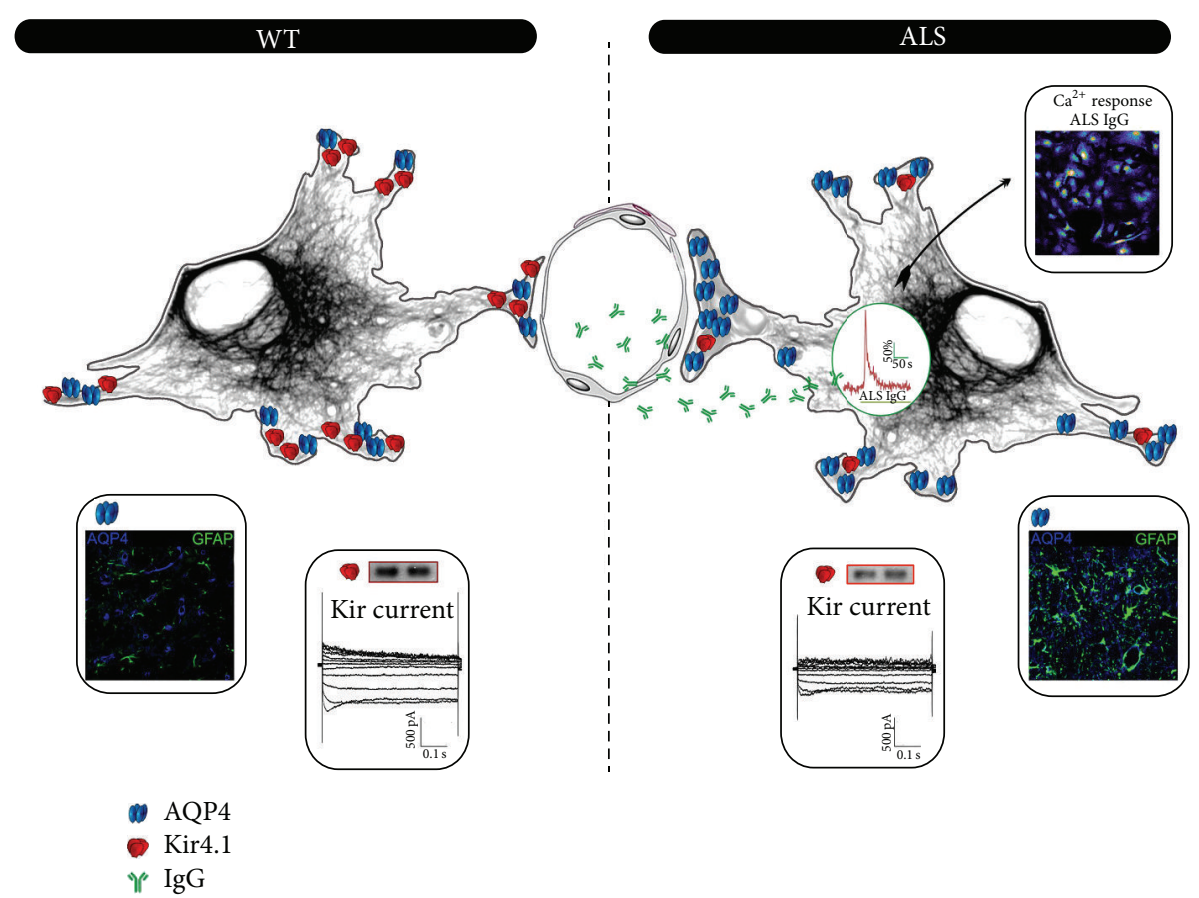

FIGURE 1: The molecular mechanism of ALS pathology in astrocytes. WT astrocyte (left) contains a balanced ratio of Kir4.1 and AQP4 channels in its cell membrane that also forms the endfeet around the endothelial layer of the blood vessel (center). ALS astrocyte (right) shows a misbalance of channel molecules with an abundance of AQP4 over Kir4.1. This causes swelling of endfeet and affects the BBB that becomes leaky for immune factors such as immunoglobulins (IgGs) that are known to cause intracellular calcium spikes (red trace and the pseudocolor image of a $\mathrm{Ca}^{2+}$-sensitive dye at the peak of the response in cultured astrocytes) and may start excitotoxic processes in situ. Colored panels in the bottom illustrate immunocytochemistry of AQP4 and GFAP in WT versus ALS astrocytes in the rat brain while the panels with traces illustrate whole-cell Kir currents from WT versus ALS astrocytes in culture and two examples of respective Western blot bands for Kir protein.

$[65,67]$. Moreover, in our studies increased AQP4 and reduced Kir4.1 expression in ALS were confirmed in cultured cortical astrocytes prepared from newborn SOD1 (G93A) rats [65]. These findings thus suggested novel molecular biomarkers for ALS and were in agreement with the changes observed in the brain tissue of symptomatic ALS rats. Our more detailed investigation revealed that functional properties of Kir4.1 channels were impaired in cultured ALS astrocytes. Thus, Kir currents isolated by addition of $\mathrm{Ba}^{2+}$ or $\mathrm{Cs}^{+}$to the extracellular solution were reduced in ALS astrocytes. As also demonstrated by electrophysiology, exposure of cultured astrocytes to elevated concentrations of extracellular potassium showed reduced capacity in buffering excessive potassium ions in ALS [65]. Furthermore, it is known that increased extracellular potassium concentration causes death of motor neurons in vitro [67]. Decreased ability of ALS astrocytes to take excessive potassium ions released after neuronal activity could lead to a disturbance of ion balance in the vicinity of motor neurons and subsequently to their degeneration.

\section{Conclusion}

Neuroinflammation is particularly prominent in ALS. It was shown that ALS IgG along with immune T cells could enter the brain parenchyma via a hampered $\mathrm{BBB}$ thus affecting neurons as well as astrocytes. Thus ALS IgG could augment excitotoxicity by stimulating transmitter release in neurons, intracellular calcium transients in neurons, and glia and vesicle trafficking/exocytosis in astrocytes. This may also confirm ALS IgG as a potential humoral marker of the disease. On the other hand it has been shown that the $\mathrm{BBB}$ compromise may lie in the misbalance of expression of the two membrane channels of the astrocytic endfeet, also possible ALS biomarkers, Kir4.1 and AQP4.

\section{Conflict of Interests}

The authors declare that there is no conflict of interests regarding the publication of this paper.

\section{Acknowledgment}

The work presented in this review was supported by the MESTD National Grant III41005.

\section{References}

[1] R. G. Miller, J. D. Mitchell, and D. H. Moore, "Riluzole for amyotrophic lateral sclerosis (ALS)/motor neuron disease (MND)," Cochrane Database of Systematic Reviews, no. 3, Article ID CD001447, 2012. 
[2] M. E. Gurney, H. Pu, A. Y. Chiu et al., "Motor neuron degeneration in mice that express a human $\mathrm{Cu}, \mathrm{Zn}$ superoxide dismutase mutation," Science, vol. 264, no. 5166, pp. 1772-1775, 1994.

[3] D. S. Howland, J. Liu, Y. She et al., "Focal loss of the glutamate transporter EAAT2 in a transgenic rat model of SOD1 mutantmediated amyotrophic lateral sclerosis (ALS)," Proceedings of the National Academy of Sciences of the United States of America, vol. 99, no. 3, pp. 1604-1609, 2002.

[4] T. J. Kwiatkowski Jr., D. A. Bosco, A. L. LeClerc et al., "Mutations in the FUS/TLS gene on chromosome 16 cause familial amyotrophic lateral sclerosis," Science, vol. 323, no. 5918, pp. 12051208, 2009.

[5] J. Sreedharan, I. P. Blair, V. B. Tripathi et al., "TDP-43 mutations in familial and sporadic amyotrophic lateral sclerosis," Science, vol. 319, no. 5870, pp. 1668-1672, 2008.

[6] J. Grosskreutz, L. van den Bosch, and B. U. Keller, "Calcium dysregulation in amyotrophic lateral sclerosis," Cell Calcium, vol. 47, no. 2, pp. 165-174, 2010.

[7] E. Foran and D. Trotti, "Glutamate transporters and the excitotoxic path to motor neuron degeneration in amyotrophic lateral sclerosis," Antioxidants and Redox Signaling, vol. 11, no. 7, pp. 1587-1602, 2009.

[8] P. van Damme, M. Dewil, W. Robberecht, and L. van den Bosch, "Excitotoxicity and amyotrophic lateral sclerosis," Neurodegenerative Diseases, vol. 2, no. 3-4, pp. 147-159, 2006.

[9] L. van den Bosch, P. van Damme, E. Bogaert, and W. Robberecht, "The role of excitotoxicity in the pathogenesis of amyotrophic lateral sclerosis," Biochimica et Biophysica ActaMolecular Basis of Disease, vol. 1762, no. 11-12, pp. 1068-1082, 2006.

[10] L. H. Barbeito, M. Pehar, P. Cassina et al., "A role for astrocytes in motor neuron loss in amyotrophic lateral sclerosis," Brain Research Reviews, vol. 47, no. 1-3, pp. 263-274, 2004.

[11] S. Garbuzova-Davis, S. Saporta, E. Haller et al., "Evidence of compromised blood-spinal cord barrier in early and late symptomatic SOD1 mice modeling ALS," PLoS ONE, vol. 2, no. 11, article e1205, 2007.

[12] C. Nicaise, D. Mitrecic, P. Demetter et al., "Impaired bloodbrain and blood-spinal cord barriers in mutant SOD1-linked ALS rat," Brain Research, vol. 1301, pp. 152-162, 2009.

[13] S. E. Nwaobi, E. Lin, S. R. Peramsetty et al., "DNA methylation functions as a critical regulator of Kir4. 1 expression during CNS development," Glia, vol. 62, no. 3, pp. 411-427, 2014.

[14] H. Kawamat, S. K. Ng, N. Diaz et al., "Abnormal intracellular calcium signaling and SNARE-dependent exocytosis contributes to SOD1G93A astrocyte-mediated toxicity in amyotrophic lateral sclerosis," The Journal of Neuroscience, vol. 34, no. 6, pp. 2331-2348, 2014.

[15] K. Meyer, L. Ferraiuolo, C. J. Miranda et al., "Direct conversion of patient fibroblasts demonstrates non-cell autonomous toxicity of astrocytes to motor neurons in familial and sporadic ALS," Proceedings of the National Academy of Sciences of the United States of America, vol. 111, no. 2, pp. 829-832, 2014.

[16] S. K. Pirooznia, V. L. Dawson, and T. M. Dawson, "Motor neuron death in ALS: programmed by astrocytes?" Neuron, vol. 81, no. 5, pp. 961-963, 2014.

[17] J. Tong, C. Huang, F. Bi et al., "Expression of ALS-linked TDP43 mutant in astrocytes causes non-cell-autonomous motor neuron death in rats," The EMBO Journal, vol. 32, no. 13, pp. 1917-1926, 2013.

[18] M. Gandelman, H. Peluffo, J. S. Beckman, P. Cassina, and L. Barbeito, "Extracellular ATP and the P2X7 receptor in astrocytemediated motor neuron death: Implications for amyotrophic lateral sclerosis," Journal of Neuroinflammation, vol. 7, article 33, 2013.

[19] T. Philips and W. Robberecht, "Neuroinflammation in amyotrophic lateral sclerosis: role of glial activation in motor neuron disease," The Lancet Neurology, vol. 10, no. 3, pp. 253-263, 2011.

[20] P. A. McCombe and R. D. Henderson, "The role of immune and inflammatory mechanisms in ALS," Current Molecular Medicine, vol. 11, no. 3, pp. 246-254, 2011.

[21] P. R. Andjus, D. Bataveljić, G. Vanhoutte et al., "In vivo morphological changes in animal models of amyotrophic lateral sclerosis and Alzheimer's-like disease: MRI approach," Anatomical Record, vol. 292, no. 12, pp. 1882-1892, 2009.

[22] M. R. Turner, R. Bowser, L. Bruijn et al., "Mechanisms, models and biomarkers in amyotrophic lateral sclerosis," Amyotrophic Lateral Sclerosis and Frontotemporal Degeneration, vol. 14, supplement 1, pp. 19-32, 2013.

[23] M. B. A. Oldstone, C. B. Wilson, L. H. Perrin, and F. H. Norris Jr., "Evidence for immune complex formation in patients with amyotrophic lateral sclerosis," The Lancet, vol. 2, no. 7978, pp. 169-172, 1976.

[24] T. G. Tachovsky, R. P. Lisak, and H. Koprowski, "Circulating immune complexes in multiple sclerosis and other neurological diseases," The Lancet, vol. 2, no. 7993, pp. 997-999, 1976.

[25] H. Bartfeld, C. Dham, and H. Donnenfeld, "Immunological profile of amyotrophic lateral sclerosis patients and their cell mediated immune responses to viral and CNS antigens," Clinical and Experimental Immunology, vol. 48, no. 1, pp. 137-146, 1982.

[26] S. Apostolski, J. Nikolić, C. Bugarski-Prokopljević et al., "Serum and CSF immunological findings in ALS," Acta Neurologica Scandinavica, vol. 83, no. 2, pp. 96-98, 1991.

[27] I. A. Saleh, T. Zesiewicz, Y. Xie et al., "Evaluation of humoral immune response in adaptive immunity in ALS patients during disease progression," Journal of Neuroimmunology, vol. 215, no. 1-2, pp. 96-101, 2009.

[28] L. Provinciali, M. A. Laurenzi, L. Vesprini et al., "Immunity assessment in the early stages of amyotrophic lateral sclerosis: a study of virus antibodies and lymphocyte subsets," Acta Neurologica Scandinavica, vol. 78, no. 6, pp. 449-454, 1988.

[29] F. Duarte, S. Binet, L. Lacomblez, P. Bouche, J.-L. Preud'homme, and V. Meininger, "Quantitative analysis of monoclonal immunoglobulins in serum of patients with amyotrophic lateral sclerosis," Journal of the Neurological Sciences, vol. 104, no. 1, pp. 88-91, 1991.

[30] M. E. Westarp, P. Bartmann, and H. Kornhuber, "Immunoglobulin-G isotype changes in human sporadic amyotrophic lateral sclerosis (ALS)," Neuroscience Letters, vol. 173, no. 1-2, pp. 124126, 1994.

[31] H. J. Willison, A. M. Chancellor, J. Veitch, P. G. E. Kennedy, and C. P. Warlow, "Immunoglobulin G subclasses are normal in motor neuron disease," Journal of the Neurological Sciences, vol. 124, no. 1, pp. 115-116, 1994.

[32] R. Zhang, R. Gascon, R. G. Miller et al., "Evidence for systemic immune system alterations in sporadic amyotrophic lateral 
sclerosis (sALS)," Journal of Neuroimmunology, vol. 159, no. 12, pp. 215-224, 2005.

[33] S. H. Appel, J. I. Engelhardt, J. Garcia, and E. Stefani, "Immunoglobulins from animal models of motor neuron disease and from human amyotrophic lateral sclerosis patients passively transfer physiological abnormalities to the neuromuscular junction," Proceedings of the National Academy of Sciences of the United States of America, vol. 88, no. 2, pp. 647-651, 1991.

[34] A. H. Pullen, M. Demestre, R. S. Howard, and R. W. Orrell, "Passive transfer of purified IgG from patients with amyotrophic lateral sclerosis to mice results in degeneration of motor neurons accompanied by $\mathrm{Ca}^{2+}$ enhancement," Acta Neuropathologica, vol. 107, no. 1, pp. 35-46, 2004.

[35] M. E. Alexianu, A. H. Mohamed, R. G. Smith, L. V. Colom, and S. H. Appel, "Apoptotic cell death of a hybrid motoneuron cell line induced by immunoglobulins from patients with amyotrophic lateral sclerosis," Journal of Neurochemistry, vol. 63, no. 6, pp. 2365-2368, 1994.

[36] F. H. Yi, C. Lautrette, C. Vermot-Desroches et al., "In vitro induction of neuronal apoptosis by anti-Fas antibodycontaining sera from amyotrophic lateral sclerosis patients," Journal of Neuroimmunology, vol. 109, no. 2, pp. 211-220, 2000.

[37] M. Demestre, A. Pullen, R. W. Orrell, and M. Orth, "ALSIgG-induced selective motor neurone apoptosis in rat mixed primary spinal cord cultures," Journal of Neurochemistry, vol. 94, no. 1, pp. 268-275, 2005.

[38] O. D. Uchitel, S. H. Appel, F. Crawford, and L. Sczcupak, "Immunoglobulins from amyotrophic lateral sclerosis patients enhance spontaneous transmitter release from motor-nerve terminals," Proceedings of the National Academy of Sciences of the United States of America, vol. 85, no. 19, pp. 7371-7374, 1988.

[39] J. I. Engelhardt, L. Siklos, L. Komuves, R. G. Smith, and S. H. Appel, "Antibodies to calcium channels from ALS patients passively transferred to mice selectively increase intracellular calcium and induce ultrastructural changes in motoneurons," Synapse, vol. 20, no. 3, pp. 185-199, 1995.

[40] S. A. Fratantoni, G. Weisz, A. M. Pardal et al., "Amyotrophic lateral sclerosis IgG-treated neuromuscular junctions develop sensitivity to L-type calcium channel blocker," Muscle Nerve, vol. 23, no. 4, pp. 543-550, 2000.

[41] A. H. Pullen and P. Humphreys, "Ultrastructural analysis of spinal motoneurones from mice treated with IgG from ALS patients, healthy individuals, or disease controls," Journal of the Neurological Sciences, vol. 180, no. 1-2, pp. 35-45, 2000.

[42] M. R. Pagani, R. C. Reisin, and O. D. Uchitel, "Calcium signaling pathways mediating synaptic potentiation triggered by amyotrophic lateral sclerosis IgG in motor nerve terminals," The Journal of Neuroscience, vol. 26, no. 10, pp. 2661-2672, 2006.

[43] R. Llinas, M. Sugimori, B. D. Cherksey et al., "IgG from amyotrophic lateral sclerosis patients increases current through P-type calcium channels in mammalian cerebellar Purkinje cells and in isolated channel protein in lipid bilayer," Proceedings of the National Academy of Sciences of the United States of America, vol. 90, no. 24, pp. 11743-11747, 1993.

[44] A. B. Zhainazarov, P. Annunziata, S. Toneatto, E. Cherubini, and A. Nistri, "Serum fractions from amyotrophic lateral sclerosis patients depress voltage-activated $\mathrm{Ca}^{2+}$ currents of rat cerebellar granule cells in culture," Neuroscience Letters, vol. 172, no. 1-2, pp. 111-114, 1994.
[45] P. R. Andjus, Z. Stevic-Marinkovic, and E. Cherubini, "Immunoglobulins from motoneurone disease patients enhance glutamate release from rat hippocampal neurones in culture," The Journal of Physiology, vol. 504, no. 1, pp. 103-112, 1997.

[46] P. R. Andjus, L. Khiroug, A. Nistri et al., "ALS IgGs suppress $\left[\mathrm{Ca}^{2+}\right]_{\mathrm{i}}$ rise through $\mathrm{P} / \mathrm{Q}$-type calcium channels in central neurones in culture," Neuroreport, vol. 7, no. 12, pp. 1914-1916, 1996.

[47] L. E. Gonzalez, M. L. Kotler, L. G. Vattino et al., "Amyotrophic lateral sclerosis-immunoglobulins selectively interact with neuromuscular junctions expressing P/Q-type calcium channels," Journal of Neurochemistry, vol. 119, no. 4, pp. 826-838, 2011.

[48] B. Li, X. Liu, Z. Li et al., "Effect of ALS IgG on motor neurons in organotypic spinal cord cultures," Canadian Journal of Neurological Sciences, vol. 35, no. 2, pp. 220-225, 2008.

[49] K. Yamanaka, S. J. Chun, S. Boillee et al., "Astrocytes as determinants of disease progression in inherited amyotrophic lateral sclerosis," Nature Neuroscience, vol. 11, no. 3, pp. 251-253, 2008.

[50] S. Boillée, K. Yamanaka, C. S. Lobsiger et al., "Onset and progression in inherited ALS determined by motor neurons and microglia," Science, vol. 312, no. 5778, pp. 1389-1392, 2006.

[51] M. Stenovec, M. Milošević, V. Petrušić et al., "Amyotrophic lateral sclerosis immunoglobulins $G$ enhance the mobility of Lysotracker-labelled vesicles in cultured rat astrocytes," Acta Physiologica, vol. 203, no. 4, pp. 457-471, 2011.

[52] M. Milošević, M. Stenovec, M. Kreft et al., "Immunoglobulins $\mathrm{G}$ from patients with sporadic amyotrophic lateral sclerosis affects cytosolic $\mathrm{Ca}^{2+}$ homeostasis in cultured rat astrocytes," Cell Calcium, vol. 54, no. 1, pp. 17-25, 2013.

[53] E. B. Malarkey, Y. Ni, and V. Parpura, " $\mathrm{Ca}^{2+}$ entry through TRPC1 channels contributes to intracellular $\mathrm{Ca}^{2+}$ dynamics and consequent glutamate release from rat astrocytes," Glia, vol. 56, no. 8, pp. 821-835, 2008.

[54] B. L. Stiles, "PI-3-K and AKT: onto the mitochondria," Advanced Drug Delivery Reviews, vol. 61, no. 14, pp. 1276-1282, 2009.

[55] J. I. Engelhardt, J. Soós, I. Obál, L. Vigh, and L. Siklós, "Subcellular localization of IgG from the sera of ALS patients in the nervous system," Acta Neurologica Scandinavica, vol. 112, no. 2, pp. 126-133, 2005.

[56] Z. Zhong, R. Deane, Z. Ali et al., "ALS-causing SOD1 mutants generate vascular changes prior to motor neuron degeneration," Nature Neuroscience, vol. 11, no. 4, pp. 420-422, 2008.

[57] S. Garbuzova-Davis, E. Haller, S. Saporta, I. Kolomey, S. V. Nicosia, and P. R. Sanberg, "Ultrastructure of blood-brain barrier and blood-spinal cord barrier in SOD1 mice modeling ALS," Brain Research, vol. 1157, no. 1, pp. 126-137, 2007.

[58] S. Garbuzova-Davis, D. G. Hernandez-Ontiveros, M. C. Rodrigues et al., "Impaired blood-brain/spinal cord barrier in ALS patients," Brain Research, vol. 1469, pp. 114-128, 2012.

[59] B. V. Zlokovic, "The blood-brain barrier in health and chronic neurodegenerative disorders," Neuron, vol. 57, no. 2, pp. 178-201, 2008.

[60] S. Nielsen, E. A. Nagelhus, M. Amiry-Moghaddam et al., "Specialized membrane domains for water transport in glial cells: high-resolution immunogold cytochemistry of aquaporin-4 in rat brain," The Journal of Neuroscience, vol. 17, no. 1, pp. 171-180, 1997. 
[61] E. A. Newman, D. A. Frambach, and L. L. Odette, "Control of extracellular potassium levels by retinal glial cell $\mathrm{K}^{+}$siphoning," Science, vol. 225, no. 4667, pp. 1174-1175, 1984.

[62] E. A. Nagelhus, Y. Horio, A. Inanobe et al., "Immunogold evidence suggests that coupling of $\mathrm{K}^{+}$siphoning and water transport in rat retinal Mülller cells is mediated by a coenrichment of Kir4. 1 and AQP4 in specific membrane domains," Glia, vol. 26, no. 1, pp. 47-54, 1999.

[63] N. N. Haj-Yasein, G. F. Vindedal, M. Eilert-Olsen et al., "Glialconditional deletion of aquaporin-4 (Aqp4) reduces bloodbrain water uptake and confers barrier function on perivascular astrocyte endfeet," Proceedings of the National Academy of Sciences of the United States of America, vol. 108, no. 43, pp. 17815-17820, 2011.

[64] D. Bataveljić, N. Djogo, L. Župunski et al., "Live monitoring of brain damage in the rat model of amyotrophic lateral sclerosis," General Physiology and Biophysics, vol. 28, pp. 212-218, 2009.

[65] D. Bataveljić, L. Nikolić, M. Milosević et al., "Changes in the astrocytic aquaporin-4 and inwardly rectifying potassium channel expression in the brain of the amyotrophic lateral sclerosis SOD1(G93A) rat model," Glia, vol. 60, no. 12, pp. 19912003, 2012.

[66] C. Nicaise, M. S. Soyfoo, M. Authelet et al., "Aquaporin-4 overexpression in rat ALS model," Anatomical Record, vol. 292, no. 2, pp. 207-213, 2009.

[67] M. Kaiser, I. Maletzki, S. Hülsmann et al., "Progressive loss of a glial potassium channel $(\mathrm{KCNJ} 10)$ in the spinal cord of the SOD1 (G93A) transgenic mouse model of amyotrophic lateral sclerosis," Journal of Neurochemistry, vol. 99, no. 3, pp. 900-912, 2006. 

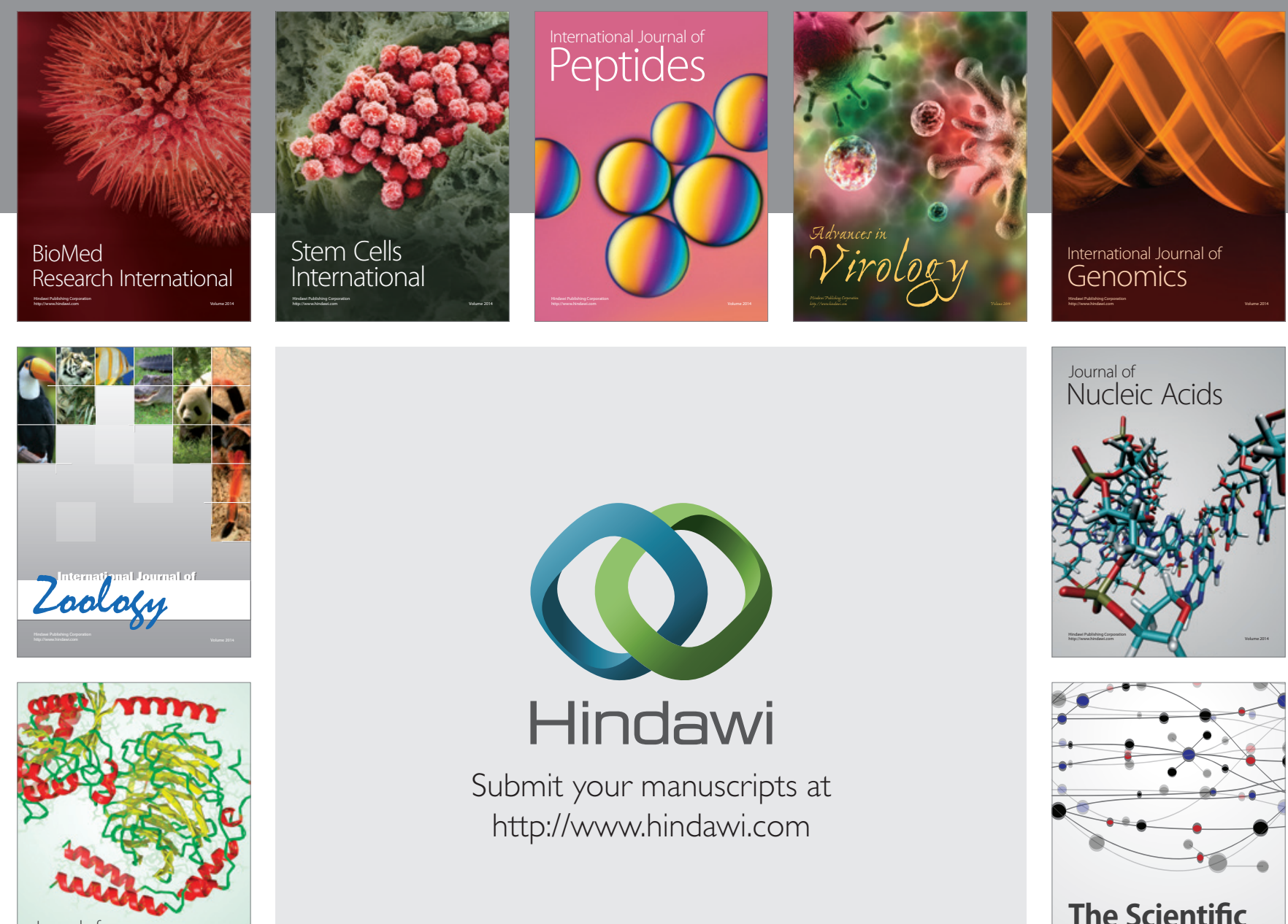

Submit your manuscripts at

http://www.hindawi.com

Journal of
Signal Transduction
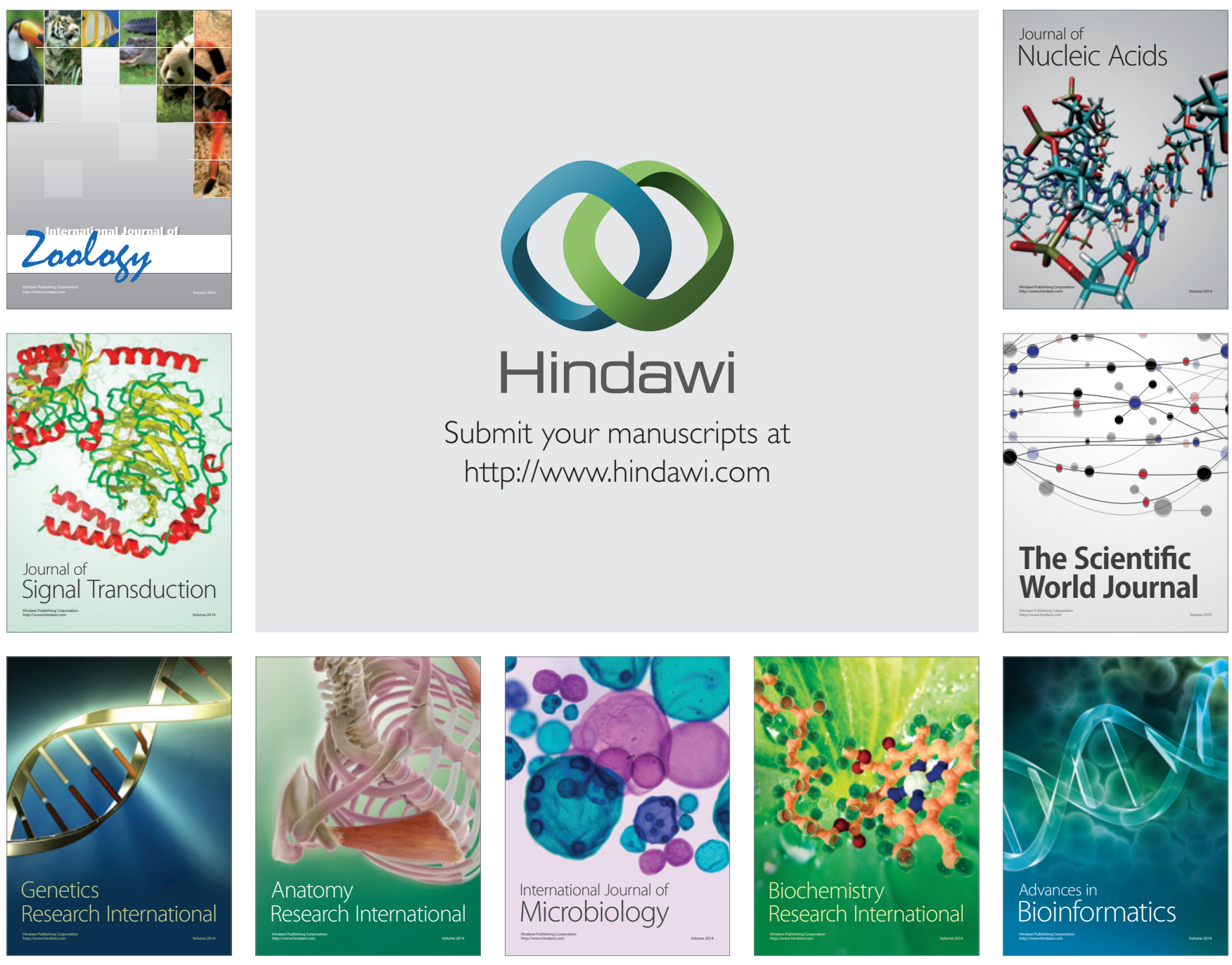

The Scientific World Journal
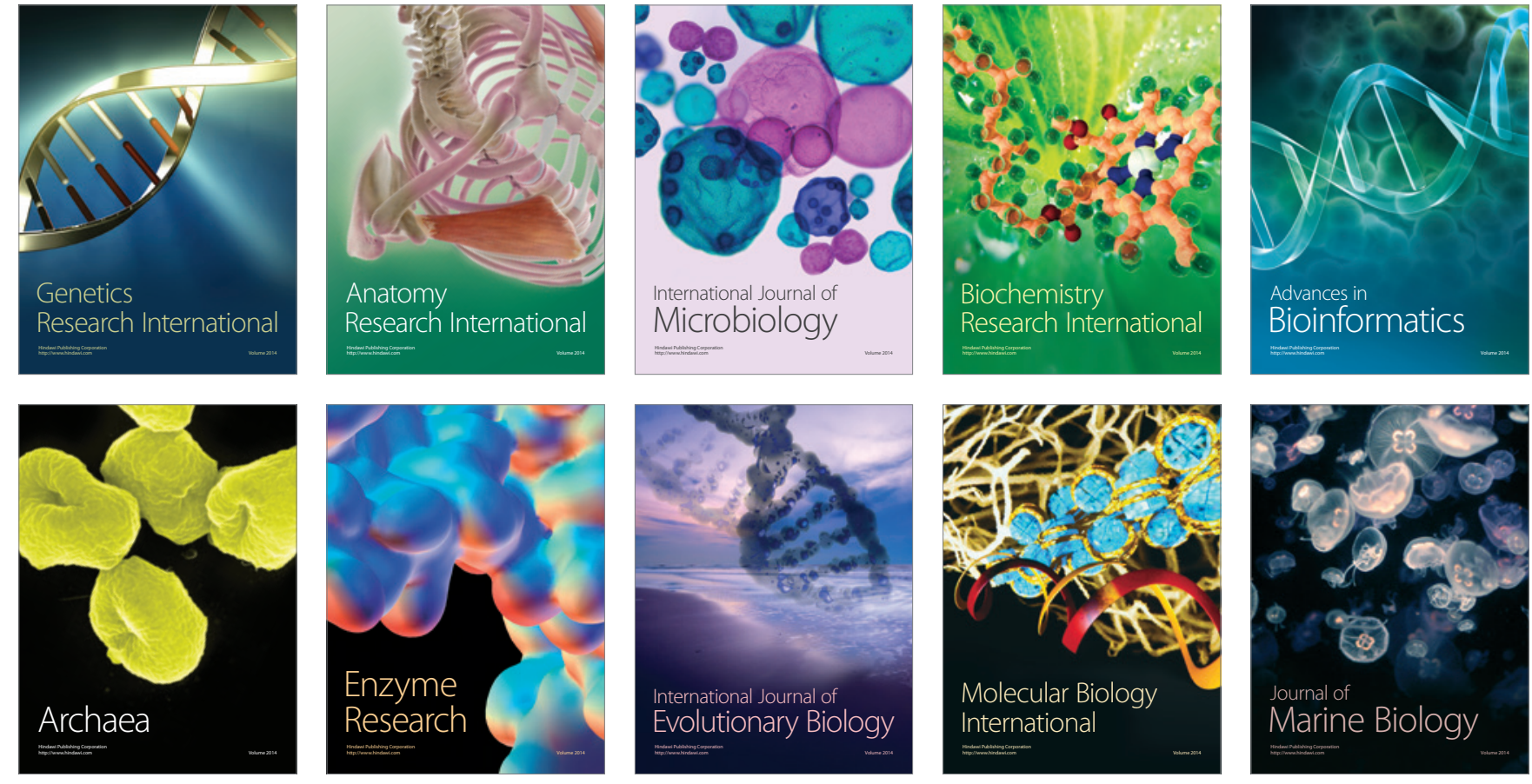